\title{
Pupil size asymmetries are modulated by an interaction between attentional load and task experience
}

\author{
Basil Wahn ${ }^{1}$, Daniel P. Ferris ${ }^{2}$, W. David Hairston ${ }^{3}$, Peter König ${ }^{1,4}$ \\ 1 Institute of Cognitive Science, University of Osnabrück, Osnabrück, Germany \\ 2 Human Neuromechanics Laboratory, School of Kinesiology, University of Michigan - \\ Ann Arbor, MI, USA \\ 3 Human Research and Engineering Directorate, U.S. Army Research Laboratory, \\ Aberdeen, MD, USA \\ 4 Department of Neurophysiology and Pathophysiology, Center of Experimental \\ Medicine, University Medical Center Hamburg-Eppendorf, Hamburg, Germany \\ * bwahn@uos.de
}

\begin{abstract}
In a recently published study [1, we investigated how human pupil sizes are modulated by task experience as well as attentional load in a visuospatial task. In particular, participants performed a multiple object tracking (MOT) task while pupil sizes were recorded using binocular eyetracking measurements. To vary the attentional load, participants performed the MOT task either tracking zero or up to five targets. To manipulate the task experience, participants performed the MOT task on three consecutive days. We found that pupil sizes systematically increased with attentional load and decreased with additional task experience. For all these analyses, we averaged across the pupil sizes for the left and right eye. However, findings of a recent study 2 have suggested that also asymmetries in pupil sizes could be related to attentional processing. Given these findings, we further analyzed our data to investigate to what extent pupil size asymmetries are modulated by attentional load and task experience. We found a significant interaction effect between these two factors. That is, on the first day of the measurements, pupil size asymmetries were not modulated by attentional load while this was the case for the second and third day of the measurements. In particular, for the second and third day, pupil size asymmetries systematically increased with attentional load, indicating that attentional processing also modulates pupil size asymmetries. Given these results, we suggest that an increase in task experience (and associated reductions in arousal) uncover modulations in pupil size asymmetries related to attentional processing that are not observable for typical arousal levels. We suggest that these modulations could be a result of right-lateralized attentional processing in the brain that in turn influences structures involved in the control of pupil sizes such as the locus coeruleus. We can exclude a number of possible alternative explanations for this effect related to our experimental setup. Yet, given the novelty of this finding and the arguably speculative explanation of the underlying mechanisms, we suggest that future studies are needed to replicate the present effect and further investigate the underlying mechanisms.
\end{abstract}




\section{Introduction}

For the past decade, researchers have investigated how modulations of pupil sizes are related to cognitive processes such as as decision-making [3 6$]$, attention [7 21], emotions 22, 23, language 24], and memory 25 28]. Moreover, changes in pupil sizes have been repeatedly associated with changes in arousal [29 32]. In these studies, modulations of pupil size were either measured in only one eye or the pupil sizes were averaged across both eyes. However, to the best of our knowledge, only one study has also investigated whether pupil size asymmetries (i.e., differences in pupil sizes between the left and right eye) are related to attentional processing 2]. In this study [2], self-rated assessments of attention ability significantly correlated with pupil size asymmetries, suggesting that also pupil size asymmetries could be modulated by attentional processing. Such a link between pupil size asymmetries and attentional processing could be explained by the right-lateralization of attentional processing in the brain 33 35] and the relation of attentional processing to structures involved in the control of pupil sizes (i.e., the locus coeruleus $14,32,36,38$ ). That is, the right-lateralization of attentional processing could systematically affect structures related to pupil size control which in turn lead to a differential modulation of the left and right pupil sizes.

In a recent study [1], we investigated the relation between attentional demands, task experience, and pupil sizes (i.e., averaged across the left and right) in a visuospatial task (i.e., a multiple object tracking (MOT) task). In this study, to vary the attentional load, participants performed the MOT task either tracking zero or up to five targets. To manipulate the task experience, participants performed the MOT task on three consecutive days. We found that pupil sizes systematically increased with attentional load and decreased with additional task experience. However, to date, it has not been investigated whether changes in attentional load also differentially affect pupil size asymmetries. In order to address this question, we further analyzed the data from our previous study to investigate to what extent pupil size asymmetries are related to changes in attentional load. Given that we measured participants on three consecutive days, we also investigated the relation of pupil size asymmetries to task experience and the interaction between the factors attentional load and task experience.

\section{Results}

For details on the methodology of the study, data preprocessing, behavioral performance in the MOT task, and results related to pupil sizes averaged across the left and right eye, we refer to our published manuscript [1].

For investigating pupil size changes that differentially affect the left and right eye, we performed the following normalization on the pre-processed data. We first subtracted the median pupil sizes of the right eye from pupil sizes of the left eye for each trial. Note, we took the median pupil size across three to nine seconds within the tracking period (i.e., while participants tracked the target objects on the computer screen) to avoid perceptual and executive confounds [1]. We then averaged these values for each participant, separately for each number of targets in the MOT task and day. We normalized the pupil size asymmetries for targets one to five by subtracting the pupil size asymmetries for the passive viewing condition and dividing the result by the pupil size in the passive viewing condition averaged across both eyes.

A descriptive overview of the normalized pupil size asymmetries can be seen in Fig 1 . In this overview, no clear modulations of pupil size asymmetries by attentional load are visible on the first day. On the second and third day, however, pupil size asymmetries appear to be differentially affected by the attentional load conditions. This observation 


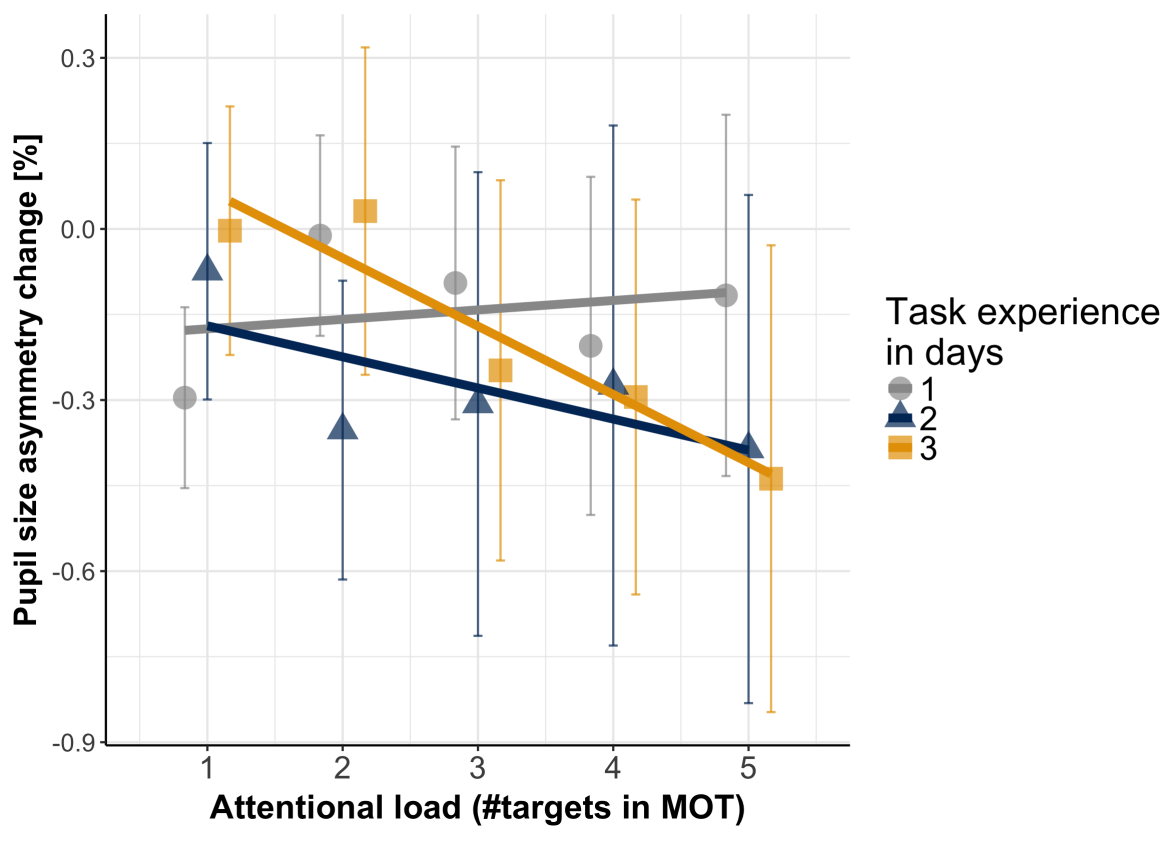

Fig 1. Pupil size asymmetries (left minus right) change relative to the passive viewing condition as a function of attentional load and task experience. Error bars are standard error of the mean. A linear regression fit is superimposed for each day.

For our inferential statistical tests, we used linear mixed models and model comparisons, starting with a baseline model in which we fitted intercepts as a random effect (i.e., intercepts were fitted for each participant individually) and used the pupil size asymmetries as the dependent variable. We then subsequently added attentional load $\left(\chi^{2}(3)=34.06, p<.001, \mathrm{BIC}=841.1\right)$ and task experience $\left(\chi^{2}(4)=85.27, p<.001\right.$, $\mathrm{BIC}=778.7)$ as fixed and random effects to the model and both yielded significant results when performing model comparisons. Adding an interaction effect between attentional load and task experience as a fixed and random effect did yield a significant model comparison, suggesting that the effect of attentional load was modulated by the effect of task experience $\left(\chi^{2}(1)=4.22, p=.040, \mathrm{BIC}=780.13\right)$. For the final model including all of these effects, we assessed the significance of the coefficients. We found a significant interaction effect $(B=-0.07, S E=0.03, p=.040)$ suggesting that with increasing task experience, the degree to which attentional load manipulations predict pupil size asymmetries increased. The coefficients for attentional load $(B=0.08, S E=$ $0.08, p=.326)$ and task experience $(B=0.25, S E=0.19, p=.197)$ by themselves were not significant.

We followed up this analysis by calculating a separate Pearson correlation coefficient for each participant for each day, correlating the attentional load with the pupil size asymmetries. For this measure, a negative correlation would indicate that the pupil size asymmetries become larger with increasing attentional load. We tested these correlation coefficients against zero using a one sample t-test - for a descriptive overview, see Fig 22. Note, prior to entering the correlations in the t-test, we applied a Fisher $\mathrm{z}$-transformation. These t-tests were not significant with regard to the first day (averaged $r=-0.33, t(19)=1.24, p=.230$ ) and second day (averaged $r=-0.26, t(19)$ $=-1.60, p=.127)$ ) but were significant for the third day (averaged $r=-0.33, t(19)=$ $-2.51, p=.021)$. These results suggest that at least for the third day, pupil size 


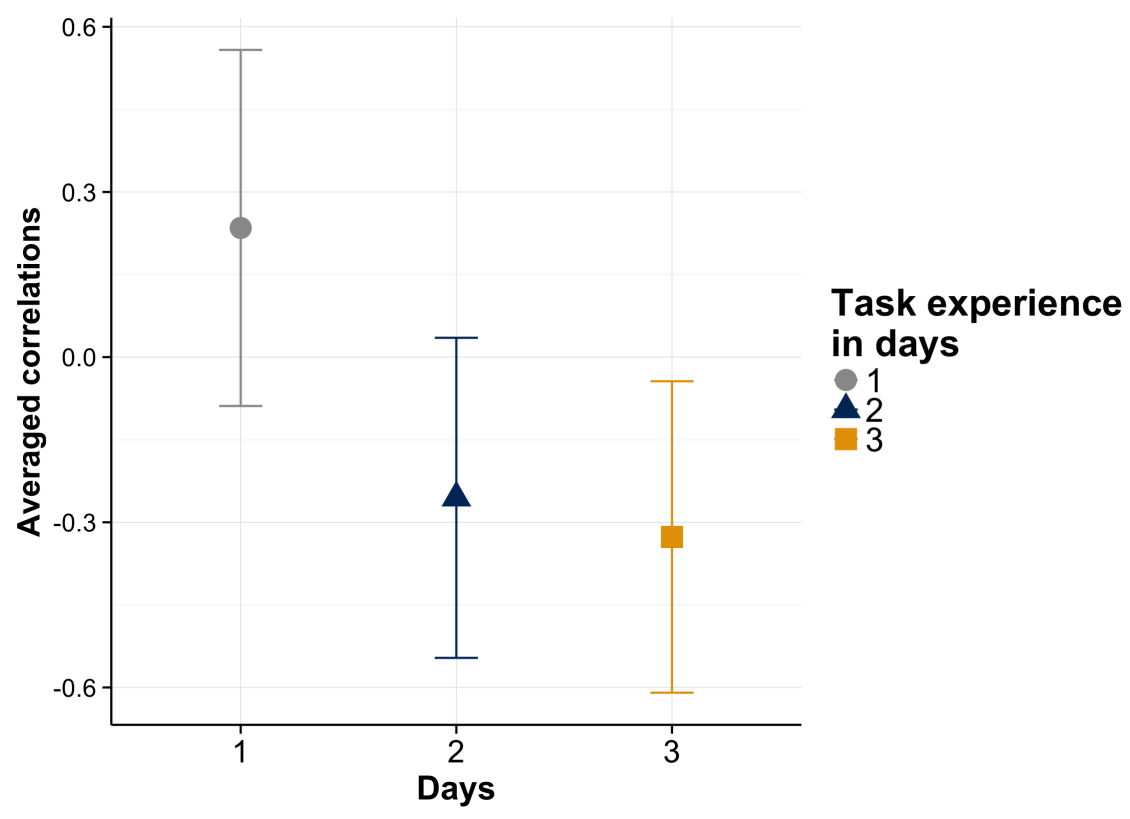

Fig 2. Averaged correlations (across participants) as a function of days. Error bars are confidence limits.

asymmetries are modulated by attentional load.

\section{Discussion}

In sum, we found that pupil size asymmetries between the left and right eye are modulated by the attentional load in a visuospatial task. However, these modulations were small, and only present on the second and third day of the measurements. Moreover, these modulations were not as fine-grained as those observed for the pupil sizes averaged across both eyes [1].

Given that these modulations were only present on the second and third day of the measurements suggests that pupil size asymmetries are additionally influenced by task experience and possibly associated reductions in arousal. That is, larger effects modulating pupil sizes possibly due to arousal could mask smaller modulations related to pupil size asymmetries and attentional processing. Modulations of pupil size asymmetries related to attentional processing may only become visible after effects of arousal are considerably reduced.

Typically, asymmetries in pupil sizes are related to pathological conditions and are assumed to be consensual otherwise. In such pathological cases, the magnitude of the asymmetry is considerably larger than reported in the present study. That is, asymmetries are clearly visible when pupil sizes are visually inspected 39.40. Here, however, the reported data suggests that pupil size asymmetries also could be modulated by an interaction of attentional load and task experience. As pointed out in the introduction section, these modulations could be a result of right-lateralized attentional processing in the brain [33 35] that in turn influences structures involved in the control of pupil sizes such as the locus coeruleus $14,32,36-38$.

As a point of note, one could suggest that modulations of pupil size asymmetries can alternatively be explained by factors related to the setup. One example is that 
participants could lean more towards the left during measurements or the left camera could be positioned more away from the eye than the right camera. We did not specifically control for these factors in our setup. However, if these factors would systematically alter results, we likely would already have observed pupil size asymmetries on the first day of the measurements. Yet, pupil size asymmetries were only present on the second and third day of the measurements. Also note, our measurement of pupil size asymmetries is a directional measure of the asymmetries. That is, we always subtracted the pupil size of the right eye from the pupil size of the left eye). We did not use an absolute measure of the asymmetries, i.e., we do not take the absolute value of the calculated differences. We suspect that any factors related to the setup that spuriously could produce pupil size asymmetries are subject to random processes (e.g., as noted above, an individual participant could lean more towards the right while another participant could lean more to the left). These random processes should likely affect absolute measures of the pupil size asymmetries. However, these processes should not systematically affect directional measures of pupil size asymmetries as it is unlikely that a large majority of participants were systematically measured differently on the first day compared to the second and third day of the measurements. Moreover, measurements for the different days for participants were often conduced on overlapping days across participants. For instance, participants who were measured on their second or last day of the measurements were measured on the same day as participants who were measured for the first time. That is, undesired changes in the setup that could have caused a modulation of pupil size asymmetries would have affected measurements in each experimental session. In summary, presently we do not have an indication that our results are based on an undesired confound in the setup.

More generally, future studies could test whether the pupil size asymmetries reported here become more pronounced when pupil sizes are measured for a more extended period of time than three consecutive days. Furthermore, it is an open question whether this effect generalizes to other types of tasks involving other forms of cognitive load. For instance, a memory task or an arithmetic task would be informative examples. Moreover, explanations on brain regions involved in pupil dilation control have primarily focused on describing general modulations of the pupil size $14,32,36,38$. Given these findings, future neurophysiological studies could also investigate the underlying processes that modulate pupil size asymmetries and may relate these to structural asymmetries in the brain 41, 42 .

\section{Acknowledgments}

We want to thank Lisa Steinmetz, Artur Czeszumski, and Ashima Keshava for their help with the data collection. We gratefully acknowledge the support by H2020 H2020-FETPROACT-2014 641321 - socSMCs (for BW), ERC-2010-AdG \#269716 MULTISENSE (for PK), and the Cognition and Neuroergonomics Collaborative Technology Alliance ARL W91 1NF-10-2-0022 (for DPF and WDH).

\section{References}

1. Wahn B, Ferris DP, Hairston WD, König P. Pupil Sizes Scale with Attentional Load and Task Experience in a Multiple Object Tracking Task. PLoS ONE. 2016;11(12):e0168087.

2. Poynter WD. Pupil-size asymmetry is a physiologic trait related to gender, attentional function, and personality. Laterality: Asymmetries of Body, Brain and Cognition. 2016; p. 1-17. 
3. Einhäuser W, Koch C, Carter O. Pupil dilation betrays the timing of decisions. Frontiers in human neuroscience. 2010;4:18.

4. Preuschoff K, Marius't Hart B, Einhäuser W. Pupil dilation signals surprise: evidence for noradrenaline's role in decision making. Frontiers in neuroscience. $2011 ; 5$.

5. de Gee JW, Knapen T, Donner TH. Decision-related pupil dilation reflects upcoming choice and individual bias. Proceedings of the National Academy of Sciences. 2014;111(5):E618-E625.

6. Murphy PR, Vandekerckhove J, Nieuwenhuis S. Pupil-linked arousal determines variability in perceptual decision making. PLOS Comput Biol.

2014;10(9):e1003854.

7. Porter G, Troscianko T, Gilchrist ID. Effort during visual search and counting: Insights from pupillometry. The Quarterly Journal of Experimental Psychology. 2007;60(2):211-229.

8. Geng JJ, Blumenfeld Z, Tyson TL, Minzenberg MJ. Pupil diameter reflects uncertainty in attentional selection during visual search. Frontiers in Human Neuroscience. 2015;9.

9. Zylberberg A, Oliva M, Sigman M. Pupil dilation: a fingerprint of temporal selection during the "attentional blink". Frontiers in Psychology. 2012;3.

10. Wierda SM, van Rijn H, Taatgen NA, Martens S. Pupil dilation deconvolution reveals the dynamics of attention at high temporal resolution. Proceedings of the National Academy of Sciences. 2012;109(22):8456-8460.

11. Naber M, Alvarez GA, Nakayama K. Tracking the allocation of attention using human pupillary oscillations. Frontiers in Psychology. 2013;4.

12. Mathôt S, Van der Linden L, Grainger J, Vitu F. The pupillary light response reveals the focus of covert visual attention. PLoS ONE. 2013;8(10):e78168.

13. Mathôt S, Melmi JB, van der Linden L, Van der Stigchel S. The mind-writing pupil: A human-computer interface based on decoding of covert attention through pupillometry. PLOS ONE. 2016;11(2):e0148805.

14. Alnæs D, Sneve MH, Espeseth T, Endestad T, van de Pavert SHP, Laeng B. Pupil size signals mental effort deployed during multiple object tracking and predicts brain activity in the dorsal attention network and the locus coeruleus. Journal of Vision. 2014;14(4):1.

15. Binda P, Murray SO. Keeping a large-pupilled eye on high-level visual processing. Trends in Cognitive Sciences. 2015;19(1):1-3.

16. Wahn B, König P. Vision and Haptics Share Spatial Attentional Resources and Visuotactile Integration Is Not Affected by High Attentional Load. Multisensory Research. 2015;28(3-4):371-392.

17. Karatekin C, Couperus JW, Marcus DJ. Attention allocation in the dual-task paradigm as measured through behavioral and psychophysiological responses. Psychophysiology. 2004;41(2):175-185.

18. Lisi M, Bonato M, Zorzi M. Pupil dilation reveals top-down attentional load during spatial monitoring. Biological Psychology. 2015;112:39-45. 
19. Hoeks B, Levelt WJ. Pupillary dilation as a measure of attention: A quantitative system analysis. Behavior Research Methods, Instruments, \& Computers. 1993;25(1):16-26.

20. Kloosterman NA, Meindertsma T, Loon AM, Lamme VA, Bonneh YS, Donner TH. Pupil size tracks perceptual content and surprise. European Journal of Neuroscience. 2015;41(8):1068-1078.

21. Heinrich W. Die Aufmerksamkeit und die Funktion der Sinnesorgane. Zeitschrift für Psychologie und Physiologie der Sinnesorgane. 1896; p. 342-388.

22. Granholm E, Steinhauer SR. Pupillometric measures of cognitive and emotional processes. International Journal of Psychophysiology. 2004;52(1):1-6.

23. Vanderhasselt MA, Remue J, Ng KK, De Raedt R. The interplay between the anticipation and subsequent online processing of emotional stimuli as measured by pupillary dilatation: the role of cognitive reappraisal. Frontiers in Psychology. $2014 ; 5$.

24. Hyönä J, Tommola J, Alaja AM. Pupil dilation as a measure of processing load in simultaneous interpretation and other language tasks. The Quarterly Journal of Experimental Psychology. 1995;48(3):598-612.

25. Kahneman D, Beatty J. Pupil diameter and load on memory. Science. 1966;154(3756):1583-1585.

26. van Rijn H, Dalenberg JR, Borst JP, Sprenger SA. Pupil dilation co-varies with memory strength of individual traces in a delayed response paired-associate task. PLoS ONE. 2012;7(12):e51134.

27. Johnson EL, Singley ATM, Peckham AD, Johnson SL, Bunge SA. Task-evoked pupillometry provides a window into the development of short-term memory capacity. Frontiers in Psychology. 2014;5.

28. Causse M, Peysakhovich V, Fabre EF. High Working Memory Load Impairs Language Processing during a Simulated Piloting Task: An ERP and Pupillometry Study. Frontiers in Human Neuroscience. 2016;10.

29. Hess EH, Polt JM. Pupil size as related to interest value of visual stimuli. Science. 1960;132(3423):349-350.

30. Simms TM. Pupillary response of male and female subjects to pupillary difference in male and female picture stimuli. Perception \& Psychophysics. 1967;2(11):553-555.

31. Stanners RF, Coulter M, Sweet AW, Murphy P. The pupillary response as an indicator of arousal and cognition. Motivation and Emotion. 1979;3(4):319-340.

32. Samuels E, Szabadi E. Functional neuroanatomy of the noradrenergic locus coeruleus: Its roles in the regulation of arousal and autonomic function part I: principles of functional organisation. Current Neuropharmacology. 2008;6(3):235.

33. De Schotten MT, Dell'Acqua F, Forkel SJ, Simmons A, Vergani F, Murphy DG, et al. A lateralized brain network for visuospatial attention. Nature neuroscience. 2011;14(10):1245-1246.

34. Flöel A, Buyx A, Breitenstein C, Lohmann H, Knecht S. Hemispheric lateralization of spatial attention in right-and left-hemispheric language dominance. Behavioural brain research. 2005;158(2):269-275. 
35. Whitehead R. Right hemisphere processing superiority during sustained visual attention. Journal of Cognitive Neuroscience. 1991;3(4):329-334.

36. Gilzenrat MS, Nieuwenhuis S, Jepma M, Cohen JD. Pupil diameter tracks changes in control state predicted by the adaptive gain theory of locus coeruleus function. Cognitive, Affective, \& Behavioral Neuroscience. 2010;10(2):252-269.

37. Wang CA, Munoz DP. A circuit for pupil orienting responses: implications for cognitive modulation of pupil size. Current opinion in neurobiology. $2015 ; 33: 134-140$.

38. Rajkowski J, Majczynski H, Clayton E, Aston-Jones G. Activation of monkey locus coeruleus neurons varies with difficulty and performance in a target detection task. Journal of Neurophysiology. 2004;92(1):361-371.

39. Lam BL, Thompson HS, Corbett JJ. The prevalence of simple anisocoria. American journal of ophthalmology. 1987;104(1):69-73.

40. Lowenstein O. Alternating contraction anisocoria: a pupillary syndrome of the anterior midbrain. AMA Archives of Neurology \& Psychiatry. 1954;72(6):742-757.

41. Galaburda AM, LeMay M, Kemper TL, Geschwind N. Right-left asymmetrics in the brain. Science. 1978;199(4331):852-856.

42. Toga AW, Thompson PM. Mapping brain asymmetry. Nature Reviews Neuroscience. 2003;4(1):37-48. 